02

\title{
Оптические характеристики пиролитического углерода, вычисленные в рамках модели эффективной среды в области колебательной моды $E_{1 u}$
}

\author{
(C) А.Н. Бехтерев, А.М. Рыжов \\ Магнитогорский государственный технический университет им. Г.И. Носова, \\ 455000 Магнитогорск, Россия \\ e-mail: alexbehterev@yandex.ru
}

Поступила в редакцию 10.09.2017 г.

В окончательной редакции 18.10.2017 г.

\begin{abstract}
Проведен расчет спектральных оптических постоянных пиролитического углерода в рамках теории эффективной среды в приближении Бруггемана в области активности колебательной моды $E_{1 u}$. Показано, что число, положение, полуширина, относительная интенсивность полос селективного поглощения в ИК спектре поглощения пиролитического углерода не зависят от величины пористости исходного поглощающего объекта.
\end{abstract}

DOI: $10.21883 / O S .2018 .03 .45649 .313-17$

Традиционными объектами для молекулярного структурного анализа выступают газы, жидкости, твердые тела, обладающие интенсивным селективным (характеристическим) колебательным спектром. Анализ названных проб осуществляется на основе спектров пропускания, отражения, комбинационного рассеяния в зависимости от оптических свойств объектов и поставленных задач. Оптическая спектроскопия является прямым, неразрушающим, достаточно прецизионным, экспрессным методом структурного анализа, позволяющим определять атомно-молекулярный состав вещества, структурную организацию молекул, фононный спектр, дефекты и примеси в кристаллической решетке образцов. Необходимо отметить, что методы ИК спектроскопии (пропускание, отражение, рассеяние) в анализе колебательного спектра более информативны, чем спектры комбинационного рассеяния, которые наиболее активно изучены в научной литературе [1-3]. Они позволяют рассчитывать оптические и диэлектрические характеристики среды, анализировать объемные и поверхностные свойства в рамках одного метода без разрушения образца, производить расчеты других физических характеристик на основе полученных предварительно дисперсионных зависимостей (поляризационных, излучательных ит.д.).

При исследовании поглощающих конденсированных объектов в режиме in situ, поверхность которых незеркальна, обладает сложным микро- и макрорельефом, традиционными методами спектроскопии отражения возникают проблемы методического учета указанных факторов в количественных измерениях. С развитием теории, технических возможностей и методологии, основанной на термопластичных элементах нарушенного полного внутреннего отражения (НПВО), предложен метод решения проблемы спектроскопического изучения неплоских, незеркальных, дисперсных сильно и слабо поглощающих объектов $[1,3]$. Проблема обнаружения и идентификации мало интенсивных селективных полос поглощения в спектрах на интенсивном фоне поглощения свободных носителей заряда до настоящего времени остается актуальной $[4,5]$. В исследованиях объектов, обладающих несплошностью структуры, возникает дополнительная проблема - учет пористости, рельефа поверхности объектов на параметры колебательных состояний и на эффективные оптические характеристики системы [6-8].

В работе с помощью математического моделирования исследуются пути решения проблемы обнаружения, идентификации колебательных состояний, влияния на них несплошности объектов, проявление данных факторов в ИК спектрах поглощения, отражения и НПВО. Согласно теории классического дисперсионного анализа (КДА), величина и спектральная зависимость оптических характеристик веществ определяется вкладом свободных и валентных электронов, а также колебательными состояниями атомов в молекулах и кристаллической решетке $[9,10]$ :

$$
\begin{aligned}
n^{2}-\varkappa^{2}=1 & +4 \pi \sum_{j} \sum_{k} N_{j} e_{j} m_{j k}^{-1} f_{j k}\left(\omega_{o j}^{2}-\omega^{2}\right) \\
& \times\left\{\left(\omega_{o j}^{2}-\omega^{2}\right)^{2}+\omega^{2}\left(g_{j} / m_{j k}\right)^{2}\right\}^{-1}, \\
2 n \varkappa= & 4 \pi \sum_{j} \sum_{k} N_{j} e_{j k} m_{j k}^{-1} f_{j k}\left(g_{j} / m_{j k}\right) \omega \\
& \times\left\{\left(\omega_{o j}^{2}-\omega^{2}\right)^{2}+\omega^{2}\left(g_{j} / m_{j k}\right)^{2}\right\}^{-1},
\end{aligned}
$$

где $n, x$ - действительная и мнимая части комплексного показателя преломления, $\tilde{n}=n+i \varkappa, j-$ число подсистем частиц, колеблющихся в поле электромагнитной волны (свободные электроны, связанные электроны, молекулы ит.п.), $k$ характеризует виды частиц в подсистемах, $e_{j k}$ и $m_{j k}$ - заряд и эффективная масса колеблющихся частиц, $N_{j}-$ концентрация $j$-типа осцилляторов, 


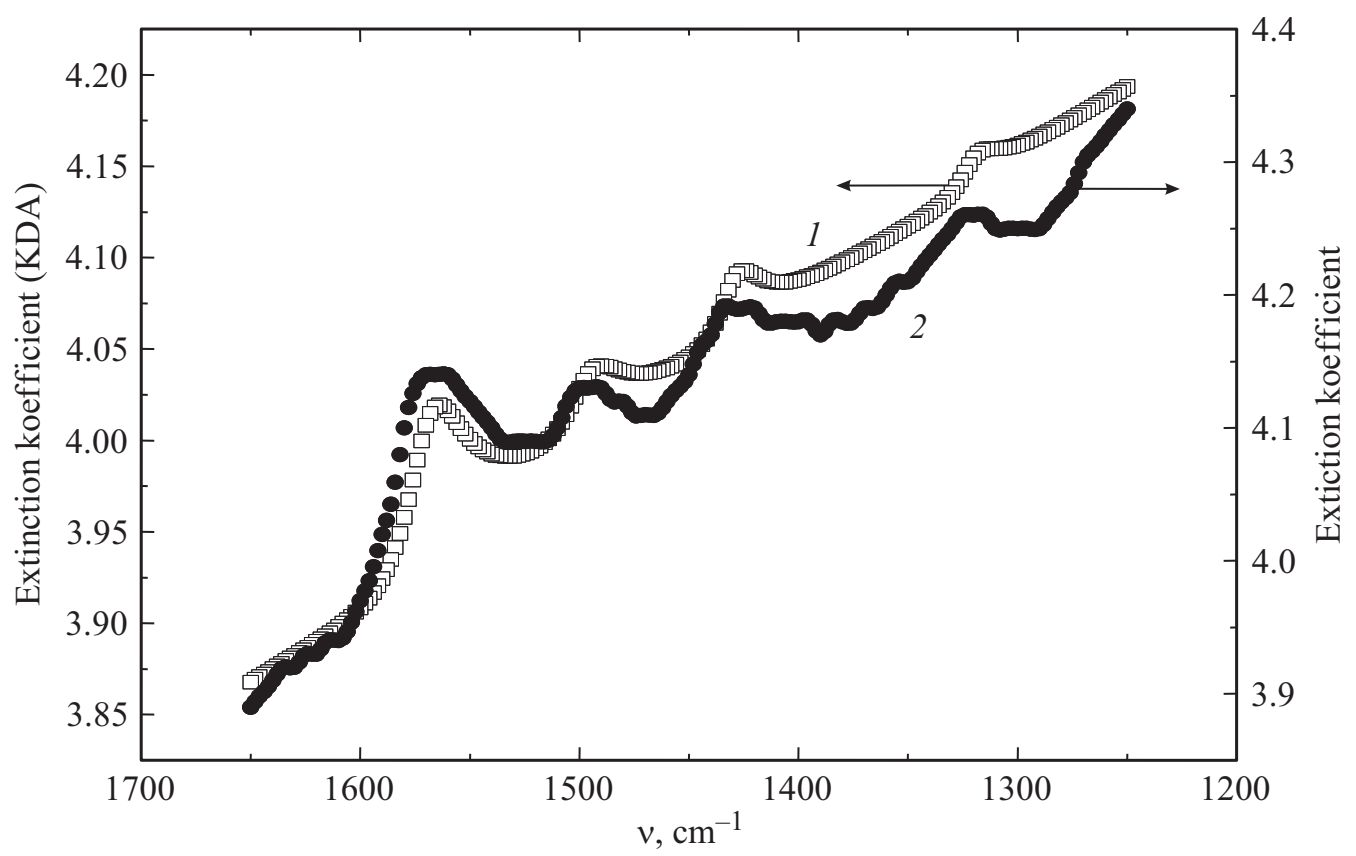

Рис. 1. Спектральная зависимость коэффициента поглощения для образца ПУ, рассчитанная по четырехосцилляторной модели (КДА, 1) и из спектра отражения (расчет по методу Крамерса-Кронига, 2).

$\omega_{o j}$ и $f_{j k}$ - собственные частоты и силы соответствующих осцилляторов, $g_{j}-$ коэффициент затухания осцилляторов типа $j, \omega-$ частота электромагнитной волны. Учитывая связь диэлектрической проницаемости с показателем преломления среды, $\tilde{\varepsilon}=\tilde{n}^{2}$, аналогичная зависимость характерна для действительной и мнимой частей диэлектрической проницаемости вещества: $\varepsilon^{\prime}, \varepsilon^{\prime \prime}$. Формулы классической теории находятся в удовлетворительном согласии с экспериментальными исследованиями оптических свойств веществ в широкой спектральной области. Из формул для оптических характеристик вещества вытекает формула Лоренц-Лорентца, разработанная до создания электромагнитной теории дисперсии и определяющая аддитивность вклада всех микрочастиц вещества, взаимодействующих с электромагнитной волной. Вдали от полос поглощения формула ЛоренцЛорентца имеет вид [9]

$$
\begin{aligned}
& n^{2}-1 / n^{2}+2= \\
& \quad=4 \pi / 3 \sum_{j} \sum_{k} N_{j} e_{j k}^{2} f_{j k}\left\{m_{j k}\left(\omega_{o j}^{2}-\omega^{2}\right)\right\}^{-1} .
\end{aligned}
$$

Для области поглощения в формуле следует рассматривать комплексную диэлектрическую проницаемость и показатель преломления $[9,10]$ :

$$
\begin{aligned}
& \tilde{n}^{2}-1 / \tilde{n}^{2}+2=4 \pi \sum_{j} N_{j} e_{j}^{2} m^{-1} \\
& \quad \times\left(\omega_{o j}^{2}-\omega^{2}+i \gamma_{j} \omega\right)^{-1}+\left(n_{o}^{2}-1\right) /\left(n_{o}^{2}+2\right),
\end{aligned}
$$

где $j$ - количество осцилляторов, $i$ - мнимая единица, $\gamma_{j}=g_{j} / m_{j}$ - приведенный коэффициент затухания колебаний. Второе слагаемое в сумме в правой части формулы определяет вклад высокочастотных составляющих в комплексный показатель преломления $\tilde{n}$. Задавая параметры осцилляторов (концентрация, собственные частоты, силы осцилляторов) и используя опорные значения коэффициента отражения или оптических постоянных, по вышеприведенным формулам (1)-(3) КДА в работе рассчитана спектральная зависимость оптических постоянных в достаточно широкой области спектра. Рассчитанные значения оптических параметров методом последовательных приближений сопоставлялись с измеренными значениями коэффициента отражения. Далее проводился последующий цикл вариации параметров.

На рис. 1 приведена спектральная зависимость показателя поглощения $\varkappa(v)$ для образца пироуглерода (ПУ) в области активности внутриплоскостных колебаний атомов углерода $\left(E_{1 u}\right)$, рассчитанная по четырехосцилляторной модели методом КДА, используя уравнения Лоренц-Лорентца. Для сравнения приведена аналогичная зависимость $\varkappa(v)$, рассчитанная по соотношениям Крамерса-Кронига из спектров отражения естественной поверхности образца ПУ с использованием термопластичных элементов [3]. Можно отметить достаточно хорошее совпадение спектров по положению и относительной интенсивности селективных полос поглощения. При расчете оптических спектров методами КДА предполагалось, что образец ПУ обладал гомогенной, изотропной структурой с равномерным распределением осцилляторов (атомов) по объему [3,11]. При этом явно количественно не учитывается соотношение ато- 


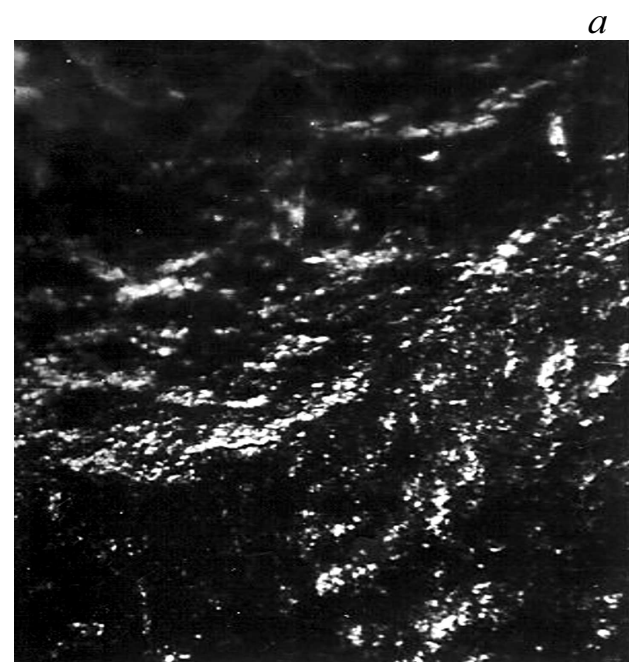

$100 \mu \mathrm{m}$

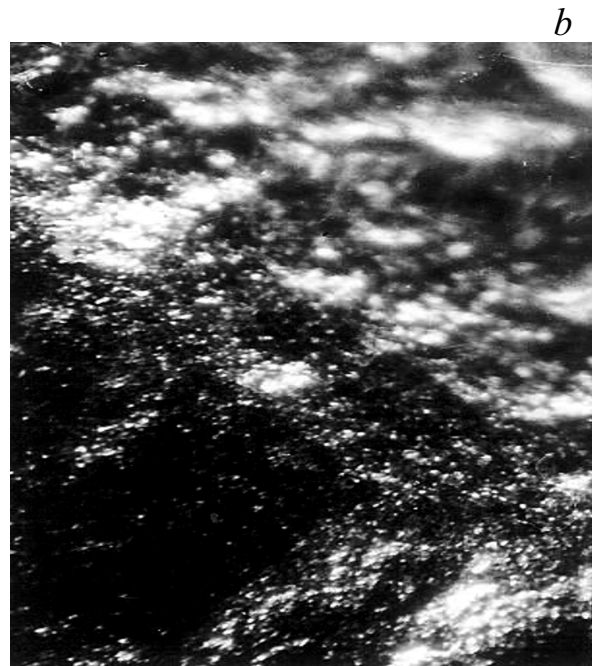

$100 \mu \mathrm{m}$

Рис. 2. Микрофотография фрагментов поверхности образца ПУ (a) и термопластичного элемента НПВО $(b)$, отнятого от образца. Увеличение $\times 500$.
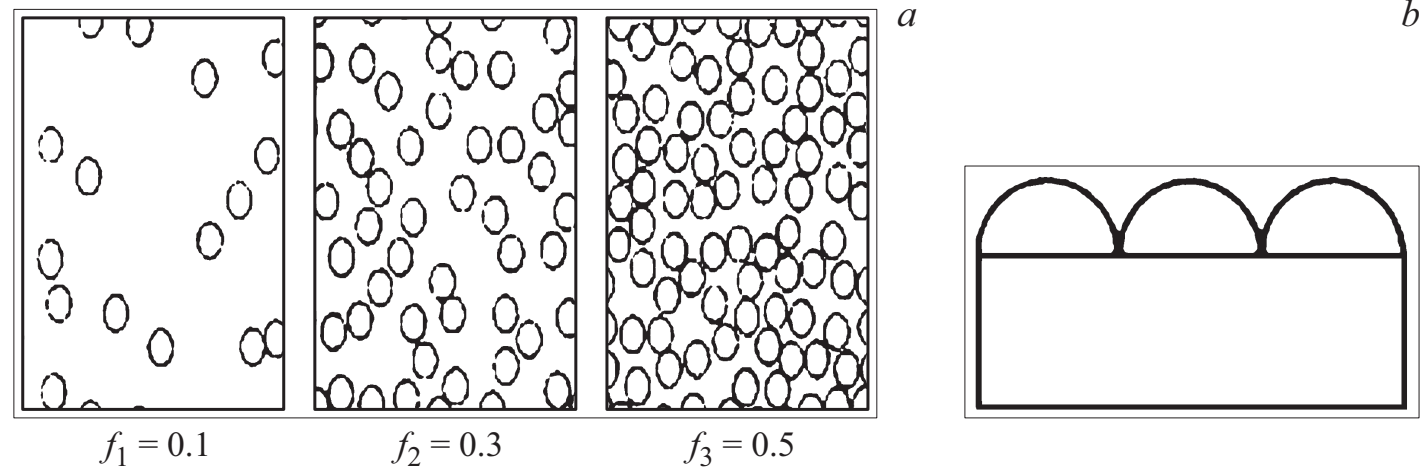

Рис. 3. Физические параметры объектов для расчета оптических характеристик несплошного объекта с различной концентрацией поглощающей компоненты $f(a)$ и микрорельефа естественной поверхности $(b)$ объекта в модели эффективной среды в приближении BR [7].

мов различного типа и их распределение в объеме системы

Дальнейшим развитием расчетных методов анализа оптических спектров была разработка теории эффективной среды для многокомпонентных сред. Здесь следует выделить в первую очередь две модели - МаксвеллГарнетта $(\mathrm{MG})$ [6,8] и Бруггемана (BR) [7,8]. Обе модели в классическом варианте были разработаны для анализа оптических характеристик двухкомпонентных сред и связывали между собой оптические и диэлектрические характеристики отдельных компонент с аналогичными параметрами композитной системы и объемными долями каждой компоненты в системе. Одна из компонент рассматривалась как диэлектрическая матрица, а другая - в качестве наполнителя с явно выраженными проводящими свойствами, где поляризованные частицы рассматривались в диэлектрической матрице [8]. B MG-модели расстояния между частицами проводящей компоненты были достаточно большими, так что можно было пренебречь их взаимодействием между собой, но учтены взаимодействия данных частиц с матрицей. B BR-модели предполагалось наличие взаимодействия фрагментов наполняющей компоненты между собой и с матрицей, что чаще выполняется для более крупных частиц или при их повышенных концентрациях

Применение моделей связано с выполнением ряда условий взаимодействия частиц с электромагнитными волнами. К основным условиям можно отнести ограничение, накладываемое на размеры частиц компонентов: средние размеры фрагментов структуры должны быть много меньшими длины волны излучения, взаимодействующего с данной средой. Это обусловлено условиями применимости макроскопических уравнений Максвелла для анализа данной системы. В работе проведены расчеты в рамках модели эффективной среды спектральной 


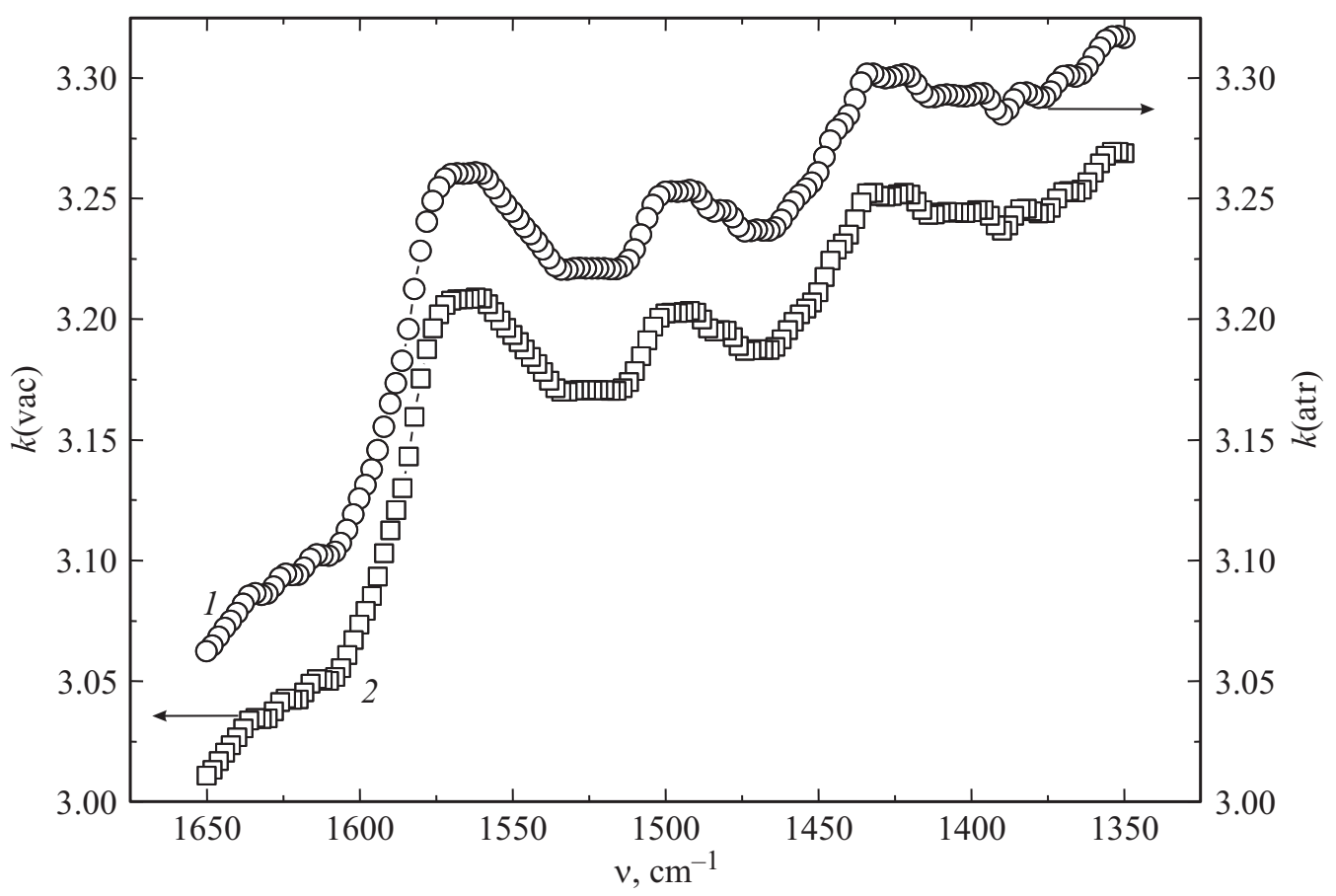

Рис. 4. Расчет оптических постоянных ПУ в модели BR с учетом включений в структуре ИКС-35 (1), вакуума (2) с объемным содержанием $f=0.25$. (ИКС-35 - техническое название литий-халькогенидных стекол в технологии термопластичных элементов НПВО [1,3]).

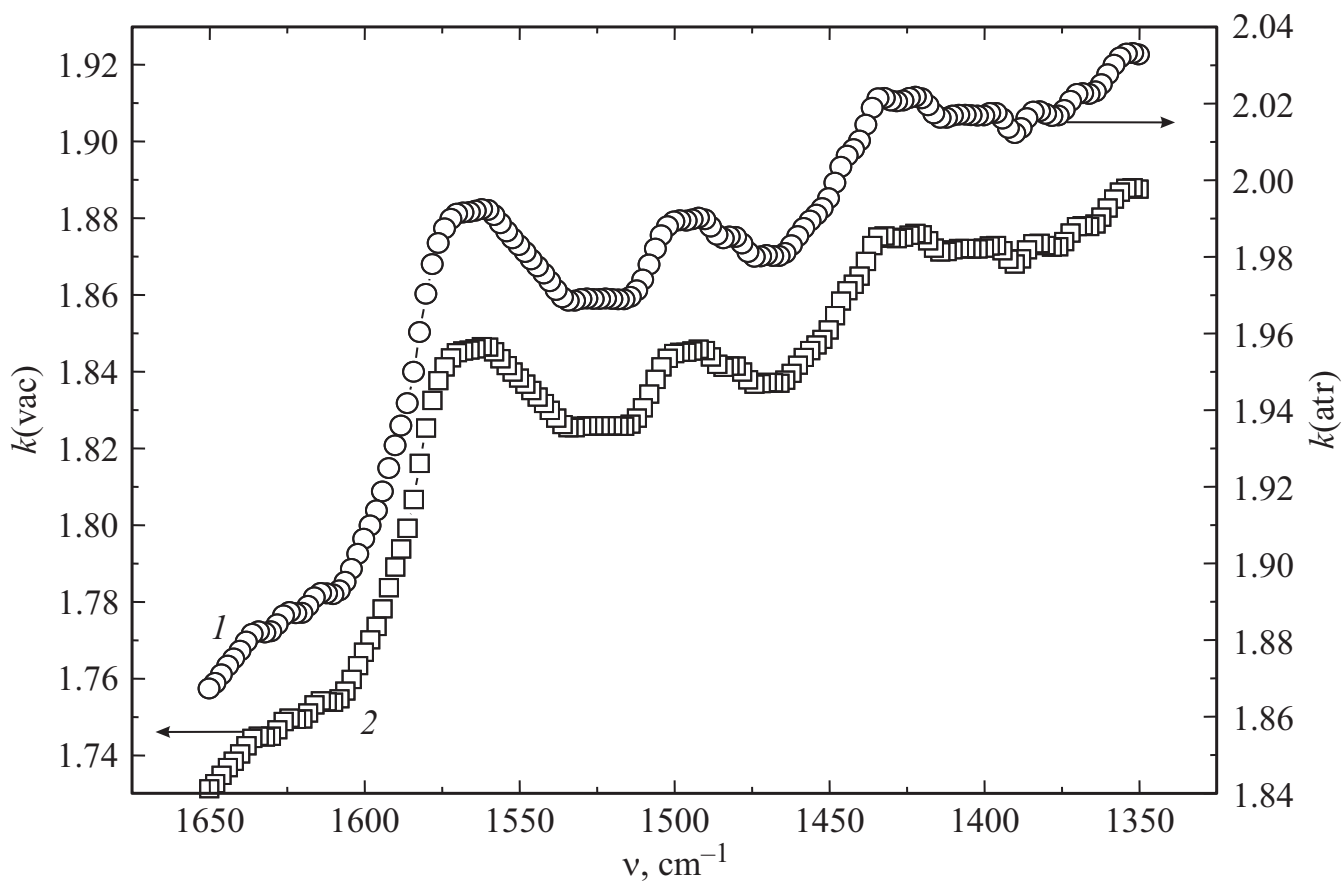

Рис. 5. Расчет оптических постоянных ПУ в модели BR с учетом включений в структуре ИКС-35 (1), вакуума (2) с объемным содержанием $f=0$.

зависимости оптических постоянных образца ПУ, обладавшего закрытой пористостью и развитым микрорельефом естественной поверхности (рис. 2) [5,11]. Макрорельеф изучаемой в режиме in situ поверхности ПУ методически учитывался при регистрации спектров НПВО с использованием термопластичных элементов [1-3]. В рамках теории эффективной среды выполнен расчет влияния микрорельефа и несплошности образца на колебательные состояния сильно поглощающих объектов. Согласно модели эффективной среды в приближениях 
MG и BR, диэлектрические характеристики системы рассчитываются соответственно по уравнениям [6-8]:

$$
\begin{gathered}
f \frac{\varepsilon_{m}-\varepsilon_{k}}{\varepsilon_{m}+2 \varepsilon_{k}}=\frac{\varepsilon_{e f}-\varepsilon_{k}}{\varepsilon_{e f}+2 \varepsilon_{k}}, \\
f \frac{\varepsilon_{m}-\varepsilon_{k}}{\varepsilon_{m}+2 \varepsilon_{k}}=\frac{(1-f)\left(\varepsilon_{e f}-\varepsilon_{k}\right)}{\varepsilon_{e f}+2 \varepsilon_{k}},
\end{gathered}
$$

где $\varepsilon_{m}, \varepsilon_{k}, \varepsilon_{e f}$ - соответственно диэлектрические постоянные матрицы, компоненты включения и композитной среды в целом, $f$ - объемная доля компоненты включения.

Данные уравнения достаточно эффективны как для анализа оптических свойств композитных систем, так и при исследовании естественной поверхности сильно поглощающих систем в случае наличия пористости или неоднородности поверхности (например, шероховатости). В последнем случае при анализе спектров отражения предполагается, что излучение проникает в объект на незначительную глубину, и в качестве диэлектрической матрицы может быть выбрана воздушная среда, а наполнителем может служить вещество объекта. Содержание поглощающей компоненты варьировалось объемной долей компоненты включения в интервале $0 \leq f \leq 0.75$ (рис. 3). На рис. 4,5 приведены типичные смоделированные спектры поглощения образцов ПУ в области проявления колебательной моды $E_{1 u}$ графита с содержанием поглощающей компоненты, наиболее близко отражающей концентрацию пор в реальных образцах конденсированного углерода $(1-40 \%)[11,12]$. Моделирование диэлектрических и оптических свойств реальной поверхности образцов ПУ на основе модели эффективной среды в приближении BR показало, что данная модель наиболее адекватно описывает оптические характеристики сильно поглощающих объектов. Пористость образцов уменьшает значения коэффициента поглощения экспериментально исследуемого объекта, не изменяя частот, полуширины и относительных интенсивностей полос поглощения, соответствующих внутриплоскостным колебательным модам и дефектам структуры (рис. 4,5). В спектре отражения явно прописываются селективные полосы, соответствующие колебательным модам $s p^{-2}$-гибридизированного углерода $\left(1580 \mathrm{~cm}^{-1)}\right.$, дефектам структуры, обусловленным деформацией гексагональных сеток $\left(1500 \mathrm{~cm}^{-1}\right)$, нарушениям трансляционной симметрии графитовой структуры $\left(1350 \mathrm{~cm}^{-1}\right)[12,13]$.

В работе исследовано влияние пористости на положение, полуширину, относительную интенсивность селективных полос поглощения в области проявления колебательных мод пиролитического углерода, относящегося к $s p^{-2}$-гибридизированному углероду методом сопоставления экспериментальных ИК спектров отражения ПУ и теоретического расчета спектров в рамках модели эффективной среды в приближении BR. Результаты сравнения спектров показали практическое отсутствие влияния пористости на названные характеристики ИК спектров отражения пироуглерода.

\section{Список литературы}

[1] Золотарев В.М. // Оптический журнал. 2000. Т. 67. № 4. C. $12-16$.

[2] Бехтерев А.Н., Золотарев В.М., Яковлев В.Б. // Опт. и спектр. 1985. Т. 59. Вып. 5. С. 1057.

[3] Бехтерев А.Н., Золотарев В.М. // Оптико-механическая промышленность. 1986. № 12. С. 41.

[4] Харрик Н. Спектроскопия внутреннего отражения. М.: Мир, 1970. 335 c.

[5] Бехтерев А.Н., Золотарев В.М. // Опт. и спектр. 2006. T. 101. № 6. C. 935.

[6] Appleyard P.G. // J. Optics A: Pure Appl. Opt. 2006. V. 8. P. 101.

[7] Forker R., Dienel T., Fritz T. // Phys. Rev. B. 2006. N 74. P. 165410(9).

[8] Ahn J.S. et al. // Phys. Rev. B. 1995. V. 52. P. 15244.

[9] Дичберн Р. Физическая оптика. М.: Наука, 1965. 632 с.

[10] Борн М. Основы оптики. М.: Наука, 1973. 719 с.

[11] Соседов В.П. $и$ др. Свойства конструкционных материалов на основе углерода. Справочник. М: Металлургия, $1975.335 \mathrm{c}$.

[12] Setton $R$. et al. Carbon Molecules and Materials. L.-NY.: Taylor and Fransis, 2002. 489 p.

[13] Бехтерев А.Н. Колебательные состояния в конденсированном углероде и наноуглероде. Магнитогорск: МаГУ, 2007. $210 \mathrm{c}$. 